\title{
Dosage de la tyramine, histamine et tryptamine dans quelques fromages français
}

\author{
par \\ P. COLONNA et J. ADDA \\ Station Centrale de Recherches Laitières et de \\ Technologie des Produits Animaux \\ I.N.R.A., C.N.R.Z. - 78350 Jouy-en-Josas
}

\section{I. - INTRODUCTION}

Le problème de la teneur des fromages en amines non volatiles (tyramine, histamine, tryptamine) reconnues comme responsables de certaines réactions physiologiques [2] a déjà suscité un certain nombre de travaux qui portent pour la plupart sur le Cheddar ou des fromages italiens $[4,5,6,13]$. Certains auteurs [15] incluent toutefois dans leur échantillon des fromages portant le nom de fromages français sans préciser s'ils sont importés ou s'il s'agit de fabrications locales, mais omettent dans la plupart des cas de donner des indications sur le degré d'affinage de ces fromages.

C'est pourquoi il a semblé intéressant d'entreprendre une étude similaire entièrement consacrée à des fromages d'origine connue et représentatifs de notre production nationale; ceci d'autant plus qu'un renouveau d'intérêt semble se dessiner au sujet de l'influence que pourraient avoir ces amines, présentes dans un certain nombre de produits alimentaires (vins, fromages, poissons [9]) sur la santé du consommateur et des conséquences que cela peut entraîner sur la consommation de fromage, soit à la suite de recommandations médicales (patients traités par des médicaments contenant des inhibiteurs de la monoamine oxydase) soit en conséquence de l'attitude de certains consommateurs tentés d'établir une corrélation entre la consommation de fromages et divers malaises (migraines) à la suite d'informations diffusées par les mass media.

\section{II - MATERIEL ET METHODES}

\section{Echantillons}

Les analyses ont porté soit sur des fromages d'âge inconnu achetés dans le commerce de détail (25 échantillons), soit sur des échan- 
tillons d'âge connu fournis par les industriels (47 échantillons). Dans ce dernier cas, une partie de ces échantillons qui avaient été prélevés dès le stade du démoulage, ont été affinés pendant un temps variable et contrôlés dans les locaux de la Laiterie Expérimentale du C.N.R.Z.

Les échantillons ont été congelés dès leur arrivée au Laboratoire et conservés à $-30^{\circ} \mathrm{C}$ jusqu'au moment de l'analyse.

\section{Méthode d'extraction}

Les amines non volatiles ont été extraites par la méthode de Cerutti [6] : $25 \mathrm{~g}$ de fromage (avec la croûte dans le cas des pâtes molles) sont mis en suspension dans $300 \mathrm{ml}$ d'acétone à l'aide d'un homogénéiseur Servall $(15 \mathrm{mn})$ [1]. L'homogénat est filtré (filtre Durieux $n^{\circ}$ 111). Le filtrat est amené à $\mathrm{pH} 1$ par apport d'HCl concentré. L'acétone est évaporée sous vide, à température inférieure à $60^{\circ} \mathrm{C}$. La matière grasse est éliminée du résidu par extraction avec $3 \times 10 \mathrm{ml}$ d'éther de pétrole (40-70). Le résidu aqueux est alcalinisé ( $\mathrm{pH}$ 10), par addition de $\mathrm{Na}_{2} \mathrm{CO}_{3}$ solide [3]. Les amines sont alors extraites sélectivement de la phase aqueuse par $3 \times 5 \mathrm{ml}$ de n-butanol.

\section{Analyse chromatographique}

On utilise des plaques de gel de silice $(20 \times 20 \mathrm{~cm} ; 250 \mu$ épaisseur), préparées en étalant un mélange de $30 \mathrm{~g}$ de gel de silice (Kieselgel G nach Stahl, Merck) et $70 \mathrm{ml}$ d'eau. Après séchage à l'air libre, les plaques sont activées à $100^{\circ} \mathrm{C}$, pendant $1 \mathrm{~h}$, puis stockées dans un dessiccateur. Elles seront réactivées par chauffage à $100^{\circ} \mathrm{C}$ pendant $30 \mathrm{mn}$ juste avant le dépôt. 5 à $50 \mu \mathrm{l}$ de la solution butanolique sont déposés à l'aide d'une micropipette (Drummond Microcaps) de $5 \mu$ l. Chaque dépôt de $5 \mu$ l est séché à l'aide d'un sèche-cheveux avant que le suivant ne soit ajouté. Pour les analyses qualitatives fines, on a utilisé deux systèmes de solvants : (butanol-acide acétiqueeau : 12-7-1) ; chloroforme - méthanol - ammoniaque 3,4 p. 100 : 2-2-1 [16]. Seul le second fut utilisé pour les analyses quantitatives. Des solutions pures de référence ont été utilisées pour l'identification.

\section{Dosage des amines}

Après développement, les plaques sont séchées $1 \mathrm{~h}$ à $55^{\circ} \mathrm{C}$ pour éliminer toute trace de solvant. On vaporise alors une solution à 0,2 p. 100 de NBD-Cl (7 Chloro - 4 nitro benzofurazan) [16] dans du méthanol jusqu'à ce que la plaque devienne translucide. On maintient ensuite les plaques pendant $24 \mathrm{~h}$ à $30^{\circ} \mathrm{C}$; puis des taches d'amines, devenues alors visibles sont grattées et mises en suspension dans $5 \mathrm{ml}$ d'acétate d'éthyle. Le mélange est agité sur un Vortex pendant $1 \mathrm{mn}$, puis centrifugé $(1000 \mathrm{~g})$ pendant $10 \mathrm{mn}$. Le surnageant est récupéré et utilisé pour les mesures de fluorescence, qui sont effectuées aux longueurs d'onde respectives d'absorption et d'excita- 
tion de $365 \mathrm{~nm}$ et $465 \mathrm{~nm}$, en utilisant des cuves de $1 \mathrm{~cm}^{2}$ de section $(1 \times 1)$ et un appareil Farrand.

Des courbes étalons ont été construites en ajoutant des quantités connues d'amines ( 0 à $2 \mathrm{mg} / \mathrm{g}$ ) à des échantillons de fromages, préalablement reconnus comme ne contenant que des quantités négligeables d'amines (Saint-Paulin).

\section{III. - RESULTATS ET DISCUSSION}

Les résultats, présentés dans les tableaux 1 à 5 , correspondent pour chaque échantillon à la moyenne des valeurs obtenues pour trois extractions indépendantes. Pour chaque type de fromage étudié on a consigné le nombre d'échantillons, l'âge et les teneurs exprimés en $\mathrm{mg} / \mathrm{g}$ de fromage brut. La notation ND signifie que l'amine est présente à une teneur inférieure au seuil de détection. qui est respectivement de $0,05,0,025$ et $0,015 \mathrm{mg} / \mathrm{g}$ pour la tyramine, la tryptamine et l'histamine. Les tableaux donnent, pour chaque fromage, la moyenne $(\mu)$ et les teneurs extrêmes rencontrées (S).

Le tableau 6 regroupe les teneurs en amines non volatiles publiées par différents auteurs.

\subsection{Fromages à pâte persillée}

Roquefort. On a regroupé les échantillons d'origines différentes en classes d'âges, classes qui se trouvent correspondre à des marques différentes, d'où sans doute une variabilité, non estimée, dont on n'a pas pu tenir compte.

La teneur moyenne en tyramine $0,70 \mathrm{mg} / \mathrm{g}$ a peu de sens à cause de la grande variabilité qui atteint 113 p. 100 . La tryptamine est présente à de très faibles concentrations : $0,33 \mathrm{mg} / \mathrm{g}$ en moyenne.

Il en est de même pour l'histamine, le plus souvent non détectable.

Si l'on ne tient pas compte du facteur « origines différentes », il semble que l'on puisse mettre en évidence une variation de la teneur en fonction de l'âge du fromage, les teneurs en amines augmentent, passent par un maximum, puis décroissent.

Fourme d'Ambert. Comparativement au Roquefort, les teneurs en tyramine et en tryptamine sont faibles $(0,55 \mathrm{mg} / \mathrm{g}$ et $0,4 \mathrm{mg} / \mathrm{g}$ respectivement). L'histamine est à peine détectable $(0,08 \mathrm{mg} / \mathrm{g})$.

Les valeurs trouvées pour ces deux fromages, dont la technologie est semblable sur bien des points, sont difficilement comparables à celles notées dans la littérature, les différents auteurs n'ayant donné aucune information sur l'âge de leur échantillon. 
TABLEAU 1. - Fromages à pâte persillée

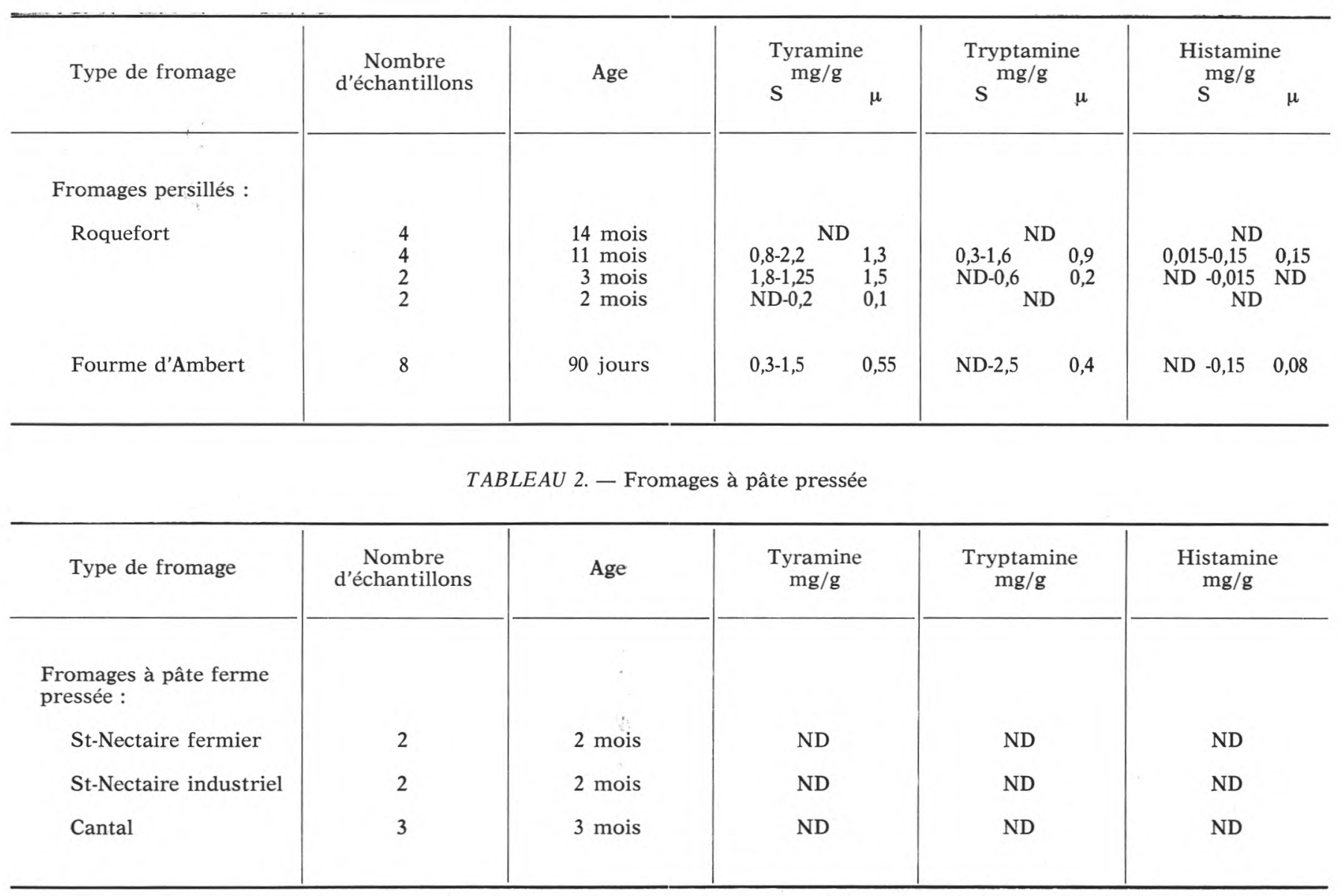


TABLEAU 3. - Fromages à pâte molle fleurie

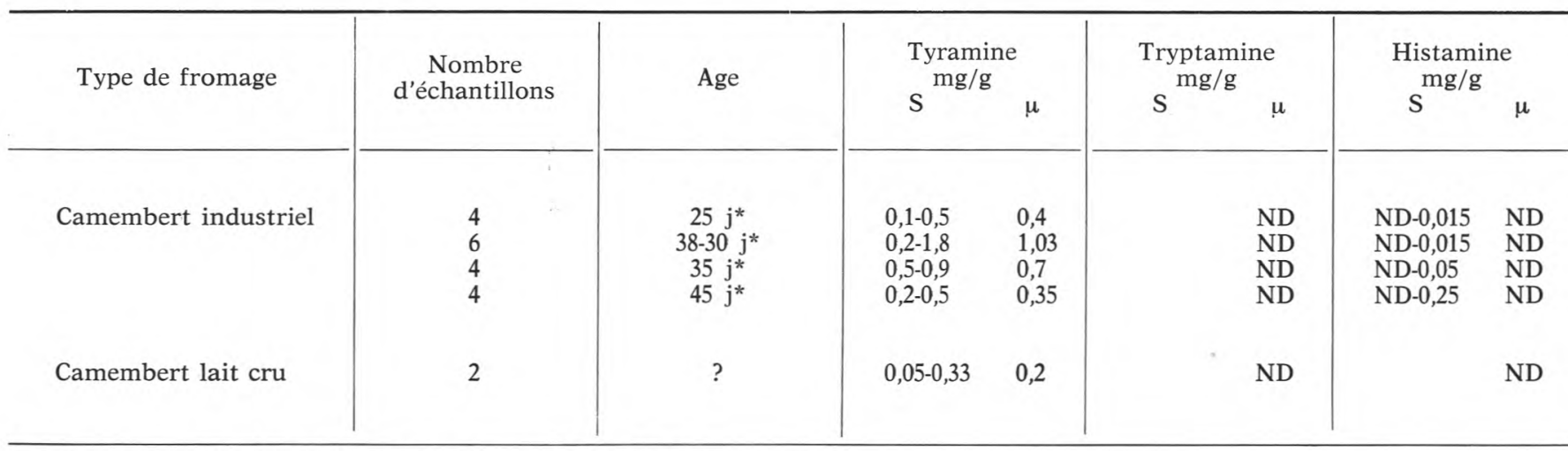

* Atfinage poursuivi à la Laiterie Expérimentale du C.N.R.Z.

TABLEAU 4. - Fromages à croûte lavée

\begin{tabular}{|c|c|c|c|c|c|c|c|}
\hline Type de fromage & $\begin{array}{c}\text { Nombre } \\
\text { d'échantillons }\end{array}$ & Age & \multicolumn{2}{|c|}{$\begin{array}{l}\text { Tyramine } \\
\mathrm{mg} / \mathrm{g}\end{array}$} & $\begin{array}{l}\text { Tryptamine } \\
\mathrm{mg} / \mathrm{g}\end{array}$ & \multicolumn{2}{|c|}{$\begin{array}{l}\text { Histamine } \\
\mathrm{mg} / \mathrm{g}\end{array}$} \\
\hline Pont L'Evêque industriel & 6 & $30-32 j^{*}$ & ND-0,26 & 0,14 & ND & ND-0,05 & ND \\
\hline Pont L'Evêque fermier & 6 & $30-32 j^{*}$ & ND-0,25 & 0,13 & ND & & ND \\
\hline Maroilles industriel & $\begin{array}{l}2 \\
1 \\
1\end{array}$ & $\begin{array}{l}7 \text { sem. } \\
8 \text { sem. } \\
9 \text { sem. }\end{array}$ & $0,15-\mathrm{ND}$ & $\begin{array}{l}0,075 \\
0,05 \\
0,20\end{array}$ & $\begin{array}{l}\text { ND } \\
\text { ND } \\
\text { ND }\end{array}$ & & $\begin{array}{l}\text { ND } \\
\text { ND } \\
\text { ND }\end{array}$ \\
\hline Maroilles fermier & 1 & 7 sem. & & 0,05 & 0,25 & & ND \\
\hline
\end{tabular}


TABLEAU 5. - Fromages à pâte cuite

\begin{tabular}{|c|c|c|c|c|c|c|c|c|}
\hline Type de fromage & $\begin{array}{c}\text { Nombre } \\
\text { d'échantillons }\end{array}$ & Age & \multicolumn{2}{|c|}{$\underset{\mathrm{mg} / \mathrm{g}}{\text { Tyramine }}$} & \multicolumn{2}{|c|}{$\begin{array}{c}\text { Tryptamine } \\
\mathrm{mg} / \mathrm{g}\end{array}$} & \multicolumn{2}{|c|}{$\begin{array}{l}\text { Histamine } \\
\mathrm{mg} / \mathrm{g}\end{array}$} \\
\hline Emmental & 2 & ? & & 0,05 & & ND & & 0,015 \\
\hline Beaufort & $\begin{array}{l}3 \\
1\end{array}$ & $\begin{array}{r}7 \text { mois } \\
18 \text { mois }\end{array}$ & & $\begin{array}{l}\text { ND } \\
0,09\end{array}$ & & $\begin{array}{l}\text { ND } \\
\text { ND }\end{array}$ & & $\begin{array}{l}\text { ND } \\
0,05\end{array}$ \\
\hline Comté & $\begin{array}{l}4 \\
1\end{array}$ & $\begin{array}{l}5,5 \text { mois } \\
15 \text { mois }\end{array}$ & $0,37-1,8$ & $\begin{array}{l}1,3 \\
0,23\end{array}$ & . & $\begin{array}{l}\text { ND } \\
\text { ND }\end{array}$ & ND-0,015 & $\begin{array}{l}0,015 \\
\text { ND }\end{array}$ \\
\hline
\end{tabular}




\section{TABLEAU 6}

Teneurs en acides aminés de différents fromages en $\mathrm{mg} / 100 \mathrm{~g}$ de fromage

\begin{tabular}{l|c|c|c|c|c|c}
\hline & Lysine & Histidine & $\begin{array}{c}\text { Trypto- } \\
\text { phane }\end{array}$ & Tyrosine & $\begin{array}{c}\text { Gamma } \\
\text { amino } \\
\text { butyrique }\end{array}$ & Arginine \\
\hline Camembert & 40 & 371 & 19 & 36 & 23 & 6 \\
Saint-Paulin & 260 & 639 & - & 137 & 29 & 149 \\
Comté & 427 & 118 & 16 & 88 & 18 & 31 \\
Bleu & & & & 35 & & \\
\hline
\end{tabular}

\subsection{Fromages à pâte ferme pressée}

On a analysé deux échantillons de Saint-Nectaire fermier, deux échantillons de Saint-Nectaire industriel et trois échantillons de Fourme du Cantal.

Les amines sont présentes en quantités inférieures au seuil de détection. Pourtant Voight [15] donne pour le Port Salut les valeurs suivantes : tyramine $0,16 \mathrm{mg} / \mathrm{g}$, tryptamine $0,16 \mathrm{mg} / \mathrm{g}$ et histamine ND. La petite taille de son échantillon (deux fromages) par rapport au nôtre (sept fromages) ne nous permet pas de comparer directement les deux séries de résultats.

\subsection{Fromage à pâte molle fleurie}

Camembert : La teneur moyenne en tyramine $0,61 \mathrm{mg} / \mathrm{g}$ est supérieure aux chiffres rencontrés dans la littérature. Pour la tryptamine, il y a concordance. L'histamine, en général non détectable, peut atteindre des valeurs de $0,25 \mathrm{mg} / \mathrm{g}$, ce qui est en accord avec les résultats de Voight.

Comme dans le cas du Roquefort, l'évolution des différentes amines en fonction du temps peut se représenter par une courbe en forme de cloche. Seuls les Camemberts fabriqués à partir de lait cru ou ceux très affinés $(45 \mathrm{j})$ donnent des résultats se rapprochant de ceux publiés dans la littérature.

\subsection{Fromages à pâte molle à croûte lavée}

Pont L'Evêque. Les teneurs en tyramine $(0,13 \mathrm{mg} / \mathrm{g})$ correspondent à celles trouvées pour le Camembert dans la littérature. $\mathrm{Ni}$ l'histamine, ni la tryptamine ne sont détectées. L'origine du fromage 
(industrielle ou fermière) ne se traduit pas par des différences notables. Au total, le Pont L'Evêque apparaît moins riche en amines non volatiles que le Camembert.

Marcilles. La petite taille de notre échantillon nous empêche de généraliser les résultats trouvés. L'histamine et la tryptamine sont toujours à la limite de détection. La tyramine est présente, à des teneurs comparables à celles trouvées dans le cas du Pont L'Evêque, autre fromage à croûte lavée.

\subsection{Fromage de chèvre}

Il n'a pas été possible de mettre d'amines en évidence sur les échantillons étudiés (Chavignolle).

\subsection{Fromages à pâte cuite}

Les trois fromages choisis se sont révélés très différents les uns des autres.

Le Beaufort, à l'âge de 7 mois, ne contient aucune amine non volatile détectable. Mais à 18 mois, apparaissent la tyramine $(0,09$ $\mathrm{mg} / \mathrm{g})$ et l'histamine $(0,05)$.

L'Emmental contient de la tyramine $(0,05 \mathrm{mg} / \mathrm{g})$ et de l'histamine $(0,015 \mathrm{mg} / \mathrm{g})$.

Le Comté, à l'âge de 5,5 mois, est riche en tyramine $(1,3 \mathrm{mg} / \mathrm{g})$. Sur un échantillon très affiné (15 mois) l'histamine cessè d'être détectable et la teneur en tyramine est faible $(0,23 \mathrm{mg} / \mathrm{g})$.

L'ensemble de ces résultats confirme l'importance de la tyramine sur la tryptamine et l'histamine. L'examen des acides aminés libres correspondants, présents dans le fromage (tab. 6) montre que les teneurs en histidine libre sont toujours supérieures aux teneurs en tyrosine. Ceci conduit à penser soit que la décarboxylation de l'histidine est moins intense que celle de la tyrosine, soit que la désamination de l'histamine se fait plus aisément que celle de la tyramine. Le mécanisme de dégradation de la tyrosine dans les fromages a été étudié par Raibaud et al. [10]. Ces auteurs montrent qu'au cours de l'affinage des protéinases actives à $\mathrm{pH}$ 5,5 libèrent la tyrosine qui sert de substrat aux décarboxylases. La tyramine ainsi produite peut ensuite être dégradée par des désaminases (également actives sur la tyrosine) en composés phénoliques neutres ou acides qui ont une part dans l'arôme du fromage. Ce mécanisme de désamination permet donc d'expliquer la décroissance de la teneur en tyramine constatée au-delà d'un certain stade d'affinage dans le cas du Roquefort et du Camembert.

On peut en second lieu remarquer que certains fromages (Roquefort, Comté) semblent à âge égal plus riches en amines que d'autres 
$T A B L E A U 7$

Teneurs en tyramine, tryptamine et histamine de différents fromages, publiées par différents auteurs

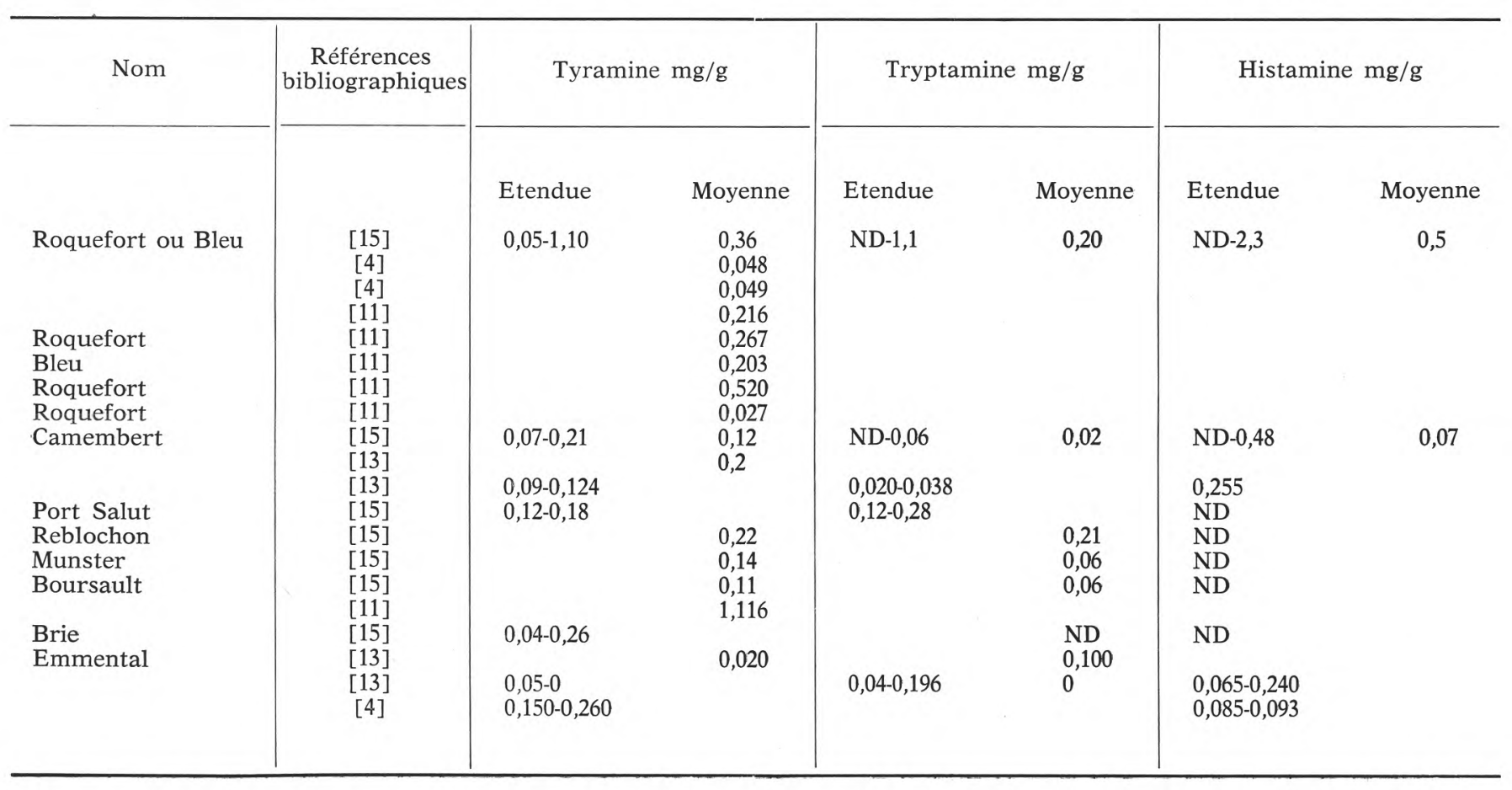


pourtant fabriqués selon une technologie voisine (Bleu, Beaufort) sans être pour autant capables d'expliquer ces différences. Enfin les pâtes molles à croûte lavée semblent systématiquement moins riches en amines que les croûtes fleuries. Dans ce dernier cas il n'a pas été possible contre toute attente de noter une différence entre fromages fabriqués à partir de lait cru et fromages fabriqués à partir de lait pasteurisé.

Enfin si l'on tente d'apprécier le risque potentiel que peut représenter l'ingestion de fromages pour les consommateurs sensibilisés thérapeutiquement, en fonction des quantités de tyramine considérées par Blackwell [2] et Voight [15] comme susceptibles de déclencher une réaction d'intolérance on est obligé de conclure à l'existence d'un tel risque. En effet, la dose maximum de tyramine supportable serait, d'après ces auteurs, de $25 \mathrm{mg}$. Il suffirait donc d'une portion de $50 \mathrm{~g}$ de la plupart des fromages examinés pour atteindre cette limite. La quantité de fromage consommable sans risque serait encore bien moindre si l'on accepte la valeur maximum de $6 \mathrm{mg}$ donnée par Harwitz (cité par Sen [11]). En ce qui concerne l'histamine, il suffirait d'en ingérer de 0,07 à $1 \mathrm{mg}$ [15] pour aboutir à une intoxication. Une portion normale de fromage fournirait dans la plupart des cas une dose supérieure au seul inférieur de 0,07 $\mathrm{mg}$.

Dans notre échantillon, seuls les fromages à pâtes pressées et le Beaufort semblent ne présenter aucun risque.

En dehors du cas extrême représenté par les patients sous l'influence d'inhibiteurs de la monoamine oxydase, le risque de voir certains malaises s'accroître à la suite de la consommation de fromages riches en amines, est plus difficile à évaluer compte tenu du manque de données publiées à ce sujet.

\section{Rés u m é}

On a étudié à l'aide d'une méthode de dosage par fluorescence les quantités de tyramine, histamine et tryptamine présentes dans soixante-treize échantillons de fromages laitiers ou fermiers, d'origine et d'âge connus. On note une diminution du taux des amines au-delà d'un certain temps d'affinage.

\section{S u m m a r y}

Tyramine, histamine and tryptamine contents of 73 cheese samples made either from raw or pasteurized milk have been studied. Tyramine content is falling down in overripened cheeses. 


\section{Références bibliographiques}

[1] Asatoon et al (1963). - Trancylcypromine and cheese. The Lancet, 5 oct.

[2] Blackwell (B.) (1965). - Tyramine in cheese related to hypertensive crises after mono amine oxidase inhibition. The Lancet, 1 mai.

[3] Block (R. J.) (1951). - Some amine acids, peptides and amines in milk and cheese. J. Dairy Sci., 34, 1.

[4] Cerutti (G.), ZAPPAvigna (R.) (1970). - Tiramina ed altre ammine pressorie negli alimenti, con particolare riferimento ai formaggi. 1. Stato della conoscenze e considerazioni. Il Latte Anno., XLV, N. 10, 707-714.

[5] Cerutti (G.), Zappavigna (R.) (1971). - Tiramina ed altre ammine pressorie alimenti, con particolare riferimento ai formaggi. 2. E. same di formaggi nazionali di vario origine. Il Latte Anno., XLV, N. 1 .

[6] Cerutti (G.) et al. (1970). - Ricerche sui formaggi fusi : composizione acidica del grasso, contenuto in polifosfati ed ammine pressorie in formaggi fusi di normale produzione. Il Latte Anno, XLIV, N. 10, 715-721.

[7] Dahlberg, Kosikowski (F. V.) (1948). The relationship of the amount and the numbers of $S$. faecalis to the intensity of flavor in american Cheddar cheese. J. Dairy Sci., 31, 305.

[8] Do Ngnoc (M.), Lenoir (J.), Choisy (C.) (1971). - Les acides aminés libres des fromages affinés de Camembert, Saint-Paulin et Gruyère de Comté. Rev. Lait. Franç., juin, 288, 447.

[9] Lovenberg (W.) (1974). - Psycho and vasoactive compounds in food substances. Agr. Food. Chem., 22 (1), 23.

[10] Raibaud (P.), Mocouot (G.), Kosikowski (F.) (1959). - Mise en évidence dans certains fromages de systèmes enzymatiques actifs sur la tyrosine. Annales de Technologie Agricole, I.N.R.A., 1, 117.

[11] SEN (N.) (1934). - Analysis and significance of tyramine in foods. J. Food Sci., 34.

[12] Silverman (G.), Kosikowski (F.) (1956), - Amines in Cheddar cheese. J. Dairy Sci., 39, 1134-1141.

[13] Spelotti (R.) (1971). - Contenuto in amine biogene di 24 formaggi italiani. Ind:Agr, 9.

[14] Voight (M.), Eitenmiller (R.) (1974). - Tyramine, histamine and tryptamine content of cheese. J. Milk Food Technol., 37.

[15] Voight (M.), Eitenmiller (R.) (1974). - Fluorescent quantitation of biologically active amines in foods with 7 chloro 4 nitro benzo-furazan. J. Food Sci., 39. 